Since the 1980s the use of percutaneous endoscopic gastrostomy (PEG) has been a safe and reliable method of enteral feeding in patients with functioning normal bowel [1]. A 74-year-old woman with Alzheimer's disease and a history of a cerebrovascular accident (CVA) was admitted after dislodgement of her PEG feeding tube. It had been placed 3 years previously using a standard pull-through technique, but had required replacement on several occasions due to dislodgement. She was found to have a large $(8 \times 9 \mathrm{~cm})$ prolapse of engorged gastric mucosa protruding from the tube tract (Figure 1). At laparotomy the prolapse was found to consist of everted full-thickness stomach wall. This was reduced and the defect was repaired in two layers (Figure 2 ). A further PEG tube was placed at a distant site. The patient made a full recovery.

Following a CVA, up to $75 \%$ of patients can require long-term PEG tube enteral feeding [2]. A number of well-documented complications have been described [3]. Cellulitis is by far the most common, followed by bleeding and leakage of gastric contents, either around the tube or intraperitoneally, causing chemical peritonitis. More unusual complications include visceral perforation with subsequent enterocutaneous and colocutaneous fistulae formation $[4,5]$. The complication described here may partly be due to the repeated track dilations to aid tube re-insertion.

\section{W. Campbell'1, G. Kirk ${ }^{1}$, S. Refsum², T. Diamond ${ }^{1}$}

${ }^{1}$ Hepatobiliary Surgical Unit, Mater Hospital Trust, Belfast, Northern Ireland

2 Surgical Unit, Belfast City Hospital Trust, Belfast, Northern Ireland.

\section{References}

${ }^{1}$ Gauderer MW. Percutaneous endoscopic gastrostomy - 20 years later: a historical perspective. J Paediatr Surg 2001; 36: 217 219

\title{
Full-Thickness Gastric Prolapse Following \\ Percutaneous Endoscopic Gastrostomy
}

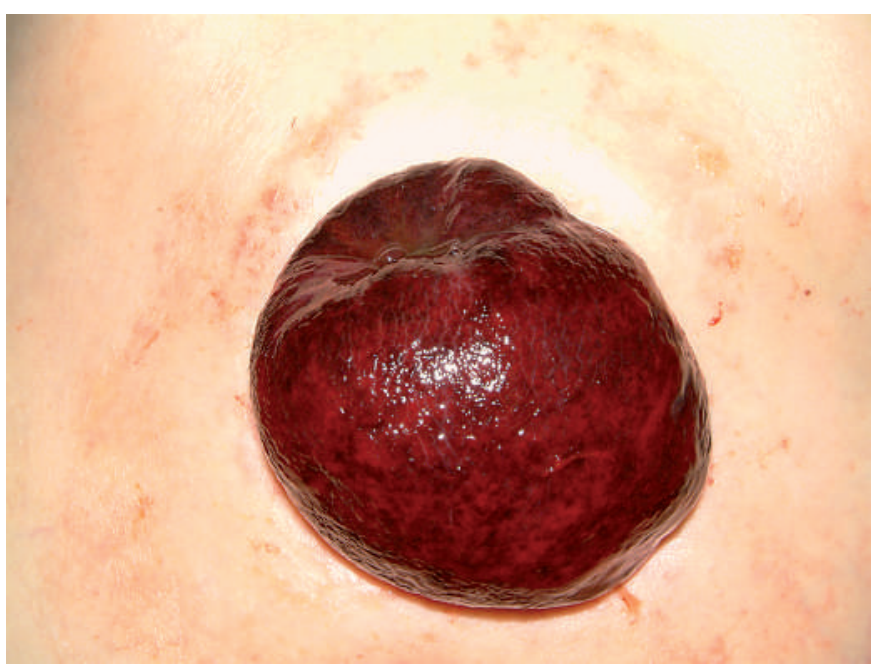

Figure 1 Full-thickness gastric mucosal prolapse.

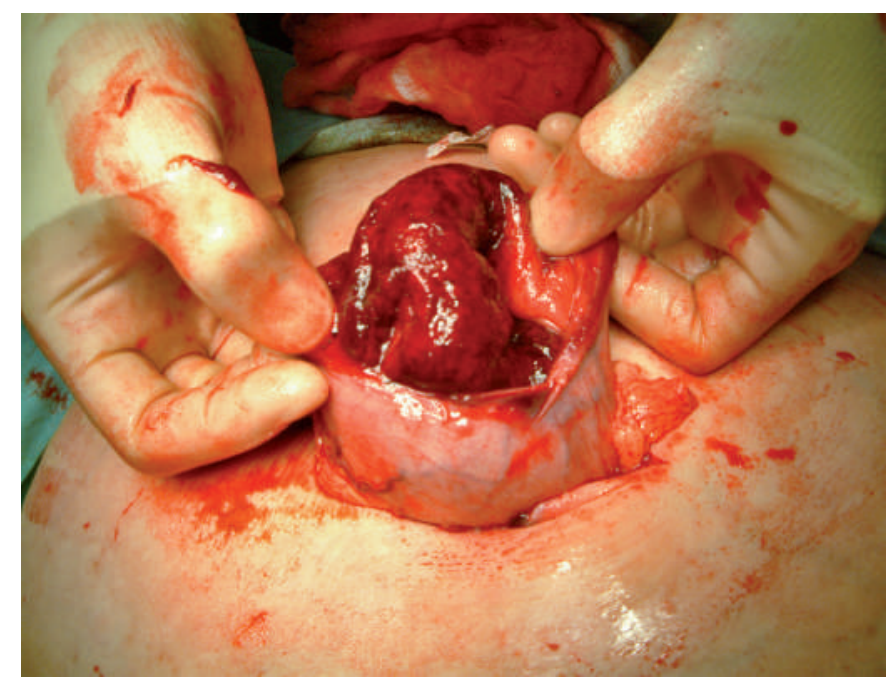

Figure 2 Repair of the prolapse.

${ }^{2}$ Ha L, Hague T. Percutaneous endoscopic gastrostomy (PEG) for enteral nutrition in patients with stroke. Scand J Gastroenterol 2003; 38: 962 - 926

3 Taylor CA, Carson DE, Ballard DJ et al. Predictors of outcome after percutaneous endoscopic gastrostomy: a community based study. Mayo Clin Proc 1992; 67: 1042 - 1049 ${ }^{4}$ Quadri AH, Puetz TR, Dindzans V et al. Enterocutaneous fistula: a rare complication of PEG tube placement. Gastrointest Endosc 2001; 53: 529-531

${ }^{5}$ Roozrokh HC, Ripepi A, Stahlfeld K. Gastrocolocutaneous fistula as a complication of PEG tube placement. Surg Endosc 2002; 16: 538-539
Corresponding Author

\section{T. Diamond, M.D.}

Mater Hospital Trust

Crumlin Road

Belfast, BT14 6AB

Ireland

Fax: $\quad+353-2890-741342$

E-mail: Tom.Diamond@mater.n-i.nhs.uk 